\title{
The Formation and Dynamics of the SMC Cluster NGC 346
}

\author{
Linda J. Smith ${ }^{1,2}$ \\ ${ }^{1}$ Space Telescope Science Institute and European Space Agency, 3700 San Martin Drive, \\ Baltimore, MD 21218, USA \\ ${ }^{2}$ Department of Physics and Astronomy, University College London, Gower St., London \\ WC1E $6 \mathrm{BT}$, UK
}

\begin{abstract}
The young resolved cluster NGC 346 in the SMC provides us with the opportunity to study the details of cluster formation and the efficiency of feedback mechanisms at low metallicity. I describe the latest results from a large-scale study of this cluster and its $\mathrm{H}$ II region N66. HST/ACS images reveal that NGC 346 is composed of a number of sub-clusters which appear to be coeval with ages of $3 \pm 1 \mathrm{Myr}$, strongly suggesting formation by the hierarchical fragmentation of a giant molecular cloud (Nota et al. 2006; Sabbi et al. 2007a). HST H $\alpha$ images show that the central cluster and the sub-clusters still contain some of their residual gas. We present high resolution spectroscopy of the ionized gas, and find that it shows little evidence for gas motions. This suggests that, at the low SMC metallicity, the cluster O star winds are not powerful enough to sweep away the residual gas. Instead, we find that stellar radiation is the dominant process shaping the interstellar environment of NGC 346.
\end{abstract}

Keywords. Galaxies: individual (SMC), galaxies: star clusters, ISM: individual (N66), ISM: H II regions, stars: formation, stars: winds, outflows

\section{Introduction}

The Small Magellanic Cloud (SMC) is an ideal laboratory to study the formation and evolution of stellar clusters at low metallicity. Its close proximity $(60 \mathrm{kpc}$; Hilditch, Howarth \& Harries 2005) means that the clusters are spatially resolved and photometry of the individual stars is well determined. During the first $\sim 1 \mathrm{Myr}$ in the evolution of a cluster, stellar winds remove the gas left over from star formation, and depending on the efficiency of star formation, this can unbind the cluster (e.g. Bastian \& Goodwin 2006). The early evolution of clusters may, however, be different at low metallicity because of the reduced stellar wind power. The investigation of this effect in recently formed clusters in the SMC is important because of the insight it provides on the formation and duration of clusters in young, low metallicity galaxies.

\section{NGC 346}

The young star cluster NGC 346 represents the most active star-forming region in the SMC. It is located towards the northern end of the SMC bar and contains at least 30 O stars (Massey, Parker \& Garmany 1989) which ionize N66, the largest H II region in the SMC. In Fig. 1, an image of NGC 346 in the light of $\mathrm{H} \alpha$ is shown. This image is taken from Nota et al. (2006) and was obtained with the Advanced Camera for Surveys (ACS) on board the Hubble Space Telescope (HST). Fig. 1 shows that even though NGC 346 is $\sim 3$ Myr old (Bouret et al. 2003), it still contains some of its residual gas. This suggests that a supernova explosion has yet to occur in the central region, and this is in agreement with the gas motions (see below) and the low diffuse X-ray flux (Nazé et al. 


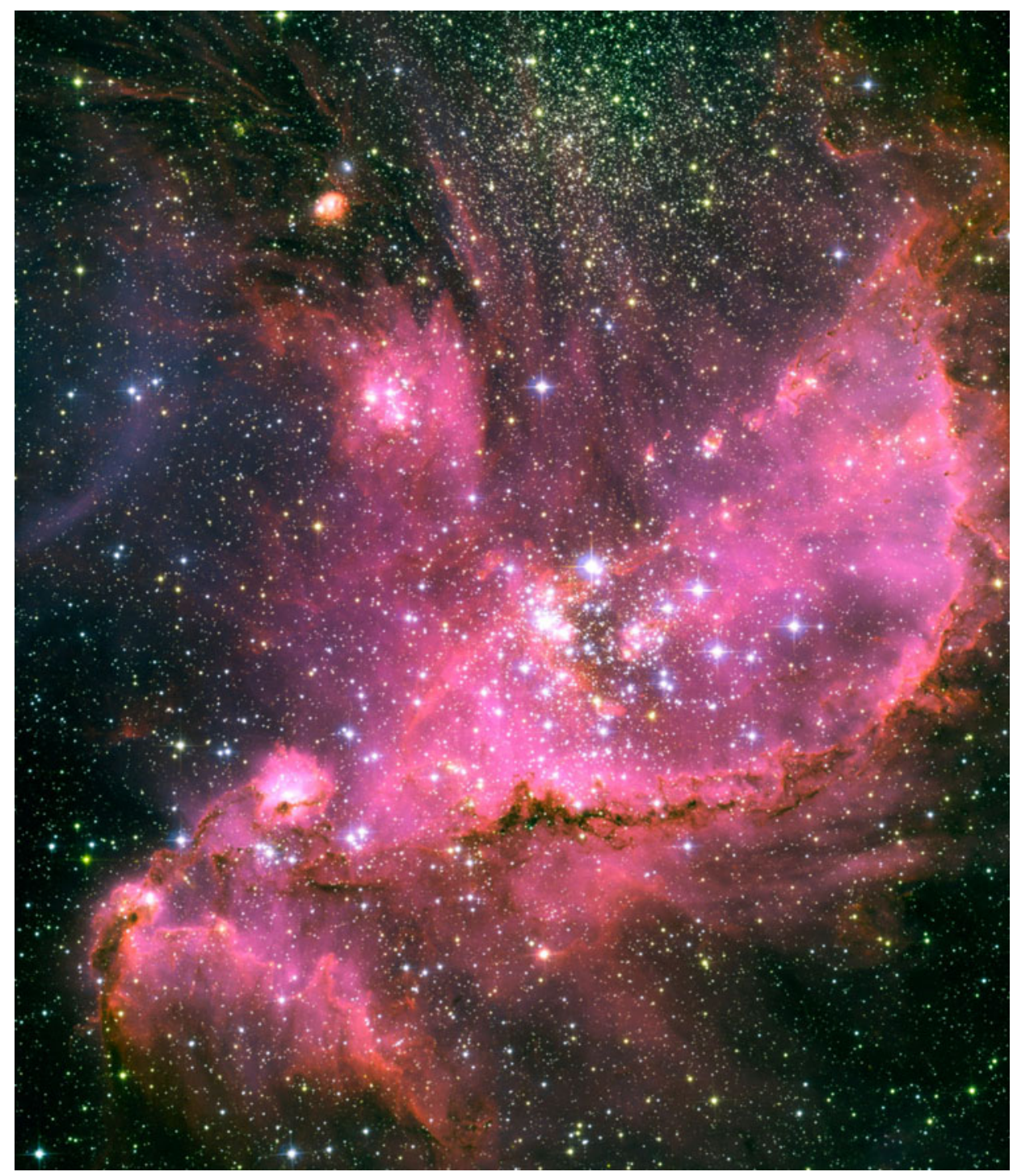

Figure 1. HST/ACS H $\alpha$ image of the SMC cluster NGC 346, covering an area of $200^{\prime \prime}$ by $200^{\prime \prime}$ (or $60 \times 60 \mathrm{pc}$ ) from Nota et al. (2006). North is up and east is to the left.

2002). There are, however, at least two supernova remnants close to NGC 346 (Ye, Turtle \& Kennicutt 1991; Reid et al. 2006).

Nota et al. (2006) and Sabbi et al. (2007a,b) present photometric analyses of NGC 346 based on HST F555W and F814W images. We find that the young stellar population of NGC 346 has an age of $3 \pm 1 \mathrm{Myr}$ and that a rich population of low mass $\left(0.6-3 \mathrm{M}_{\odot}\right)$ pre-main sequence stars also exists, that were probably formed at the same time as the central cluster.

We find that the young stellar population is not uniformly distributed within the ionized nebula. We identify at least 15 sub-clusters that differ in size and stellar content. The locations of these sub-clusters are shown in Fig. 2 which is taken from Sabbi et al. (2007a). The central cluster contains at least three sub-clusters (Sc1, 2 and 3), and has a half-mass radius of $9 \mathrm{pc}$ and a total stellar mass of $\sim$ few $\times 10^{5} \mathrm{M}_{\odot}$ (Sabbi et al. 2007b). 


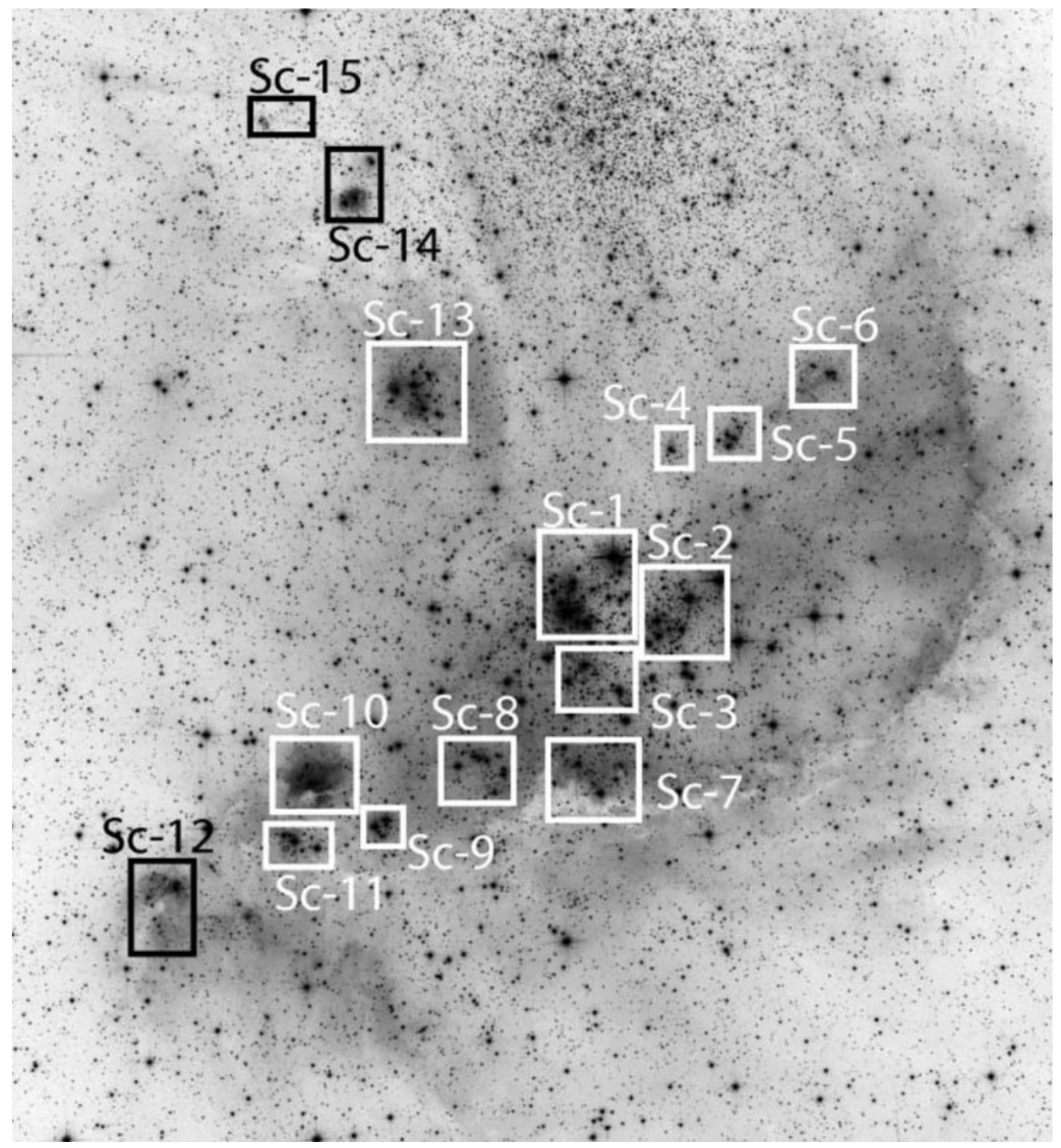

Figure 2. HST/ACS F555W image of the SMC cluster NGC 346 showing the 15 identified sub-clusters.

Within the uncertainties of the isochrone fitting process, we find that the sub-clusters are coeval with an age of $3 \pm 1 \mathrm{Myr}$. They are still embedded in nebulosity and coincide with clumps of neutral gas and CO emission (Rubio et al. 2000; Contursi et al. 2000).

The pre-main sequence stars detected in the HST images are concentrated in the centre of NGC 346 and are also present, in smaller numbers, in most of the small compact subclusters (e.g. Sc-10, 12, 13 and 14). Simon et al. (2007) have recently presented Spitzer observations of NGC 346. They find 111 embedded young stellar objects (YSOs), showing that star-formation is still ongoing in this region today. The most massive YSOs in their sample (if they are single objects) are located in the central sub-clusters, presenting strong evidence for primordial mass segregation. They also find that all but one of the 15 sub-clusters identified by Sabbi et al. (2007a) contain YSOs.

Recently, Sabbi et al. (2007b) have determined the present day mass function (PDMF) for NGC 346 from their deep HST photometry. We find that the PDMF is Salpeter $(\Gamma=-1.43 \pm 0.18)$ over the mass range of $0.8-60 \mathrm{M}_{\odot}$. The PDMF slope varies as 


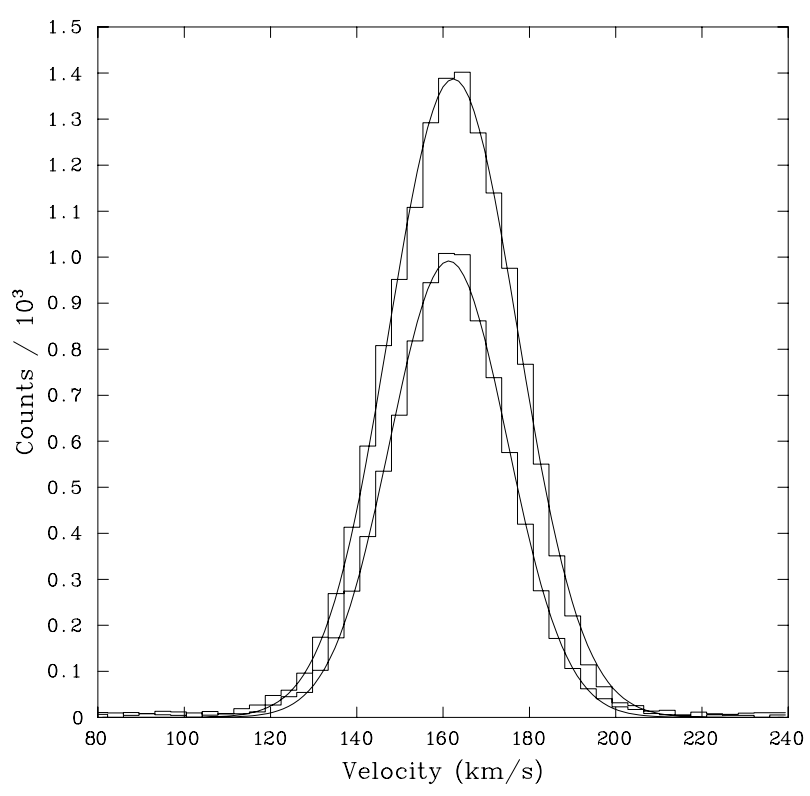

Figure 3. AAT+UCLES H $\alpha$ profiles for the slit crossing the sub-cluster Sc-6 in NGC 346. The two profiles, with Gaussian fits superimposed, represent data from the extremes of the 1 arcmin long slit, and show that the nebular gas is quiescent.

a function of the radial distance from the centre of the cluster, indicating that mass segregation, probably of primordial origin, is present.

The crossing-time from the central sub-cluster to the outer sub-clusters (Sc 6, 12 and 15) is $\sim 2 \mathrm{Myr}$ (for a sound speed of $10 \mathrm{~km} \mathrm{~s}^{-1}$ ) and thus NGC 346 is about one crossing-time old. The observed sub-cluster structure and the coevality of the sub-clusters strongly suggest that NGC 346 was formed by the collapse, and subsequent hierarchical fragmentation of a giant molecular cloud (GMC) into multiple seeds of star formation (Elmegreen 2000; Klessen \& Burkert 2000; Bonnell \& Bate 2002; Bate, Bonnell \& Bromm 2003; Bonnell, Bate \& Vine 2003). In this model, the fragmentation of the GMC is due to supersonic turbulent motions in the gas. The turbulence induces the formation of shocks, and produces filamentary structures. The chaotic nature of the turbulence results in local density enhancements in the filamentary structures. High density regions that become self-gravitating then collapse to form stars, and this happens simultaneously at different locations within the cloud (Bonnell et al. 2003).

To test these theories and to study the effect of stellar winds at the low metallicity of the SMC, we have obtained high spectral resolution observations of the ionized gas in NGC 346/N66. These data were obtained with the University College London Echelle Spectrograph (UCLES) on the Anglo-Australian Telescope (AAT), and cover the H $\alpha$ and [O III] $\lambda 5007$ emission lines at a spectral resolution of $6 \mathrm{~km} \mathrm{~s}^{-1}$. We observed a total of six slit positions across N66 covering a number of sub-clusters. Surprisingly, we find that the ionized gas is quiescent with no evidence for large-scale gas motions. In general, we find that the $\mathrm{H} \alpha$ profiles are single with a velocity dispersion of $\sim 14 \mathrm{~km} \mathrm{~s}^{-1}$ and a constant velocity along the length of each slit position (1 arc min). An example is shown in Fig. 3, where the $\mathrm{H} \alpha$ profiles for the two extremes of the slit crossing Sc-6 are plotted. Even at the centre of NGC 346 where the most massive O stars are located, we detect no significant ionized gas motions. These results strongly suggest that at the low metallicity of the SMC, stellar winds are much reduced, and the dominant form of interaction is via 
stellar radiation rather than by winds. This is in accord with Bouret et al. (2003) who measured the mass loss rates of six $\mathrm{O}$ stars within the central cluster and found that their winds are considerably weaker than their Galactic counterparts. We also find that there are very small differences $\left(\leqslant 3 \mathrm{~km} \mathrm{~s}^{-1}\right)$ in the velocities of the sub-clusters, as measured by their ionized gas motions. This suggests that if the sub-clusters merge with the main cluster in the centre, it will take at least $\sim 7 \mathrm{Myr}$.

\section{Acknowledgements}

I would like to thank and acknowledge my collaborators on the NGC 346 project: A. Nota, L. Angeretti, L. Carlson, J. Gallagher, M. Meixner, M. S. Oey, A. Pasquali, E. Sabbi, M. Sirianni, M. Tosi \& R. Walterbos.

\section{References}

Bate, M. R., Bonnell, I. A., \& Bromm, V. 2003, MNRAS 339, 577

Bastian, N. \& Goodwin, S. P. 2006, MNRAS 369, L6

Bonnell, I. A. \& Bate, M. R. 2002, MNRAS 336, 659

Bonnell, I. A., Bate, M. R., \& Vine, S. 2003, MNRAS 343, 413

Bouret, J.-C., Lanz, T., Hillier, D. J., Heap, S. R., Hubeny, I., Lennon, D. J., Smith, L. J., \& Evans, C. J. 2003, ApJ 595, 1182

Contursi, A. et al. 2000, A\& $A, 362,310$

Elmegreen, B. G. 2000, AJ 530, 227

Hilditch, R. W., Howarth, I. D., \& Harries, T. J. 2005, MNRAS 357, 304

Klessen, R. S. \& Burkert, A. 2000, ApJS 128, 287

Massey, P., Parker, J. W., \& Garmany, C. D. 1989, AJ 98, 1305

Nazé, Y., Hartwell, J. M., Stevens, I. R., Corcoran, M. F., Chu, Y.-H., Koenigsberger, G., Moffat, A. F. J., \& Niemela, V.S. 2002, ApJ 580, 225

Nota, A. et al. 2006, ApJ 640, L29

Reid, W. A., Payne, J. L., Filipović, M. D., Danforth, C. W., Jones, P. A., White, G. L., \& Staveley-Smith, L. 2006, MNRAS 367, 1379

Rubio, M., Contursi, A., Lequeux, J., Probst, R., Barbá, R., Boulanger, F., Cesarsky, D., \& Maoli, R. 2000, A\&\&A 359, 1139

Sabbi, E. et al. 2007a, AJ 133, 44

Sabbi, E. et al. 2007b, AJ in press

Simon, J. D. et al. 2007, ApJ 669, 327

Ye, T., Turtle, A. J., \& Kennicutt, R. C. 1991, MNRAS 249, 722 